\title{
Research on the Modeling of Helicopter Background Magnetic Field based on the Magnetic Dipole Array
}

\author{
Jianyang Zhao ${ }^{1, a}$, Chunsheng Lin ${ }^{1, b}$ \\ ${ }^{1}$ Department of Weaponry Engineering, Naval University of Engineering, Wuhan, 430033, China \\ aemail: zhao1986213@126.com, bemail:Ics-and-zh@163.com
}

Keywords: Magnetic Anomaly; Magnetic Dipole; Helicopter Pob; Anti-Submarine Technology

\begin{abstract}
Magnetic anomaly anti-submarine technology is very important, this paper analyzes in detail the background magnetic interference characteristics of magnetic anomaly antisubmarine helicopter platform, establishes a magnetic dipole array model of helicopter background magnetic field, points out the practical getparms methods of the model, and also puts forward the important problems to be solved in the next step.
\end{abstract}

\section{Introduction}

In future sea wars, aeronautical antisubmarine technology is indispensable. Relative to the sonar detection technology, aeronautical magnetic anomaly antisubmarine technology is a kind of passive and flexible detection and its detection precision is high[1]. The aeronautical antisubmarine platforms at present are usually fixed wing aircraft, unmanned aerial vehicles and carrier-based helicopter, etc[2,3]. Aiming at helicopter platform, to accurately detect submarine magnetic anomaly, the helicopter magnetic interference must be compensated[4]. So to build the model of helicopter background magnetic field is very important. This paper analyzes in detail the background magnetic interference characteristics of magnetic anomaly antisubmarine helicopter platform, establishes a magnetic dipole array model of helicopter background magnetic field, and gives the simulate results of the magnetic field.

\section{Analysis of helicopter background magnetic interference characteristics}

There are a lot of magnetic objects on helicopter such as electrical equipments, engine cylinder, energized cables and rotor bearing. During flight, the magnetic interference generated by these magnetic objects comprises remanent magnetism, induced magnetism and eddy current magnetism[5]. The magnetic interference strength is related to helicopter magnetism, flight attitude, heading, helicopter location and the pod position.

Geomagnetic field magnetizes those aircraft magnetic objects for a long time, forming the remanent magnetism which remains unchanged in a few days to tens of days. The induced magnetism is generated when aircraft magnetic objects are magnetized instantly by geomagnetic field, and it is relative to flight attitude and the magnetic vector of measurement point. Eddy current magnetism is produced by aircraft magnetic objects cutting geomagnetic field lines, associated with flight attitude, flight velocity, shape and size of these objects and other factors.

There are rotor wings equipped on middle and tail of helicopter, and while the wings rotating larger magnetic interference will be produced nearby. Therefore, the high resolution magnetic field measurement device should be out of helicopter rotor for a certain distance while using the measurement platform, in order to reduce the magnetic interference effect on the magnetic field measurement results, and to ensure the accuracy and resolution of magnetic field measurement.

At present aviation magnetic field measurement system is mainly hanged or trailed outside the helicopter. For the hanging system, to compensate the magnetic interference generated at the pod by helicopter, helicopter background magnetic interference model should be established and solved. 


\section{Magnetic dipole array model of helicopter background magnetic field}

According to the characteristics of helicopter magnetic field measurement system, the pod is relatively far from aircraft cabin, and the pod rope length is generally more than $20 \mathrm{~m}$.

So the magnetic field generated by aircraft at pod position can be approximately equivalent to the magnetic field generated by some magnetic dipoles which are located in the central axis of plane.

A rectangular coordinate system is established, taking a helicopter center as the origin, aircraft longitudinal axis as $\mathrm{X}$ axis while front is positive, plane transverse as $\mathrm{Y}$ axis while right is positive, and the axis perpendicular to XOY plane as $\mathrm{Z}$ axis while underneath is positive(see Fig.1).

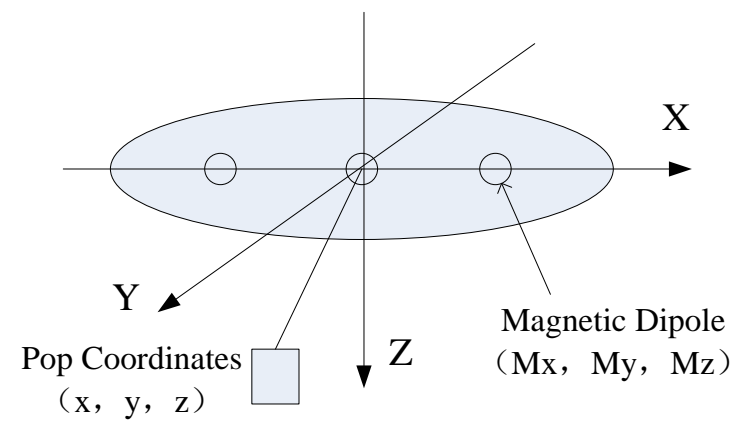

Fig.1. Helicopter magnetic dipole array model

Magnetic moment of magnetic dipoles can be expressed as follows

$\vec{M}_{i}=\left(M_{x i}, M_{y i}, M_{z i}\right) \quad i=1,2, \cdots N$

Where $\mathrm{N}$ is the amount of magnetic dipole. The magnetic moment of each dipole includes remanent magnetic moment, induced magnetic moment and eddy current magnetic moment, and the three-component magnetic moment can be expressed as follows:

$$
\left\{\begin{array}{l}
M_{x i}=P_{x i}+K_{x i}+E_{x i} \\
M_{y i}=P_{y i}+K_{y i}+E_{y i} \quad i=1,2, \cdots N \\
M_{z i}=P_{z i}+K_{z i}+E_{z i}
\end{array}\right.
$$

Where the remanent magnetic moment vector is

$\vec{P}_{i}=\left(p_{x i}, p_{y i}, p_{z i}\right) \quad i=1,2, \cdots N$

Because the intrinsic magnetic field of plane is accumulated for a long time and remains basically unchanged in a few days, the remanent magnetic moment can be regarded as a constant vector.

The induced magnetic moment can be expressed as:

$$
\left\{\begin{array}{l}
K_{x i}=k_{x i} \cdot B \cdot u_{1} \\
K_{y i}=k_{y i} \cdot B \cdot u_{2} \\
K_{z i}=k_{z i} \cdot B \cdot u_{3}
\end{array} \quad i=1,2, \cdots N\right.
$$

Where $\vec{B}$ is the geomagnetic vector and $\left(k_{x i}, k_{y i}, k_{z i}\right)$ is the induced magnetic moment coefficient, a fixed parameter, relative to geometry, quality, and permeability of magnetic material. $u_{i}=\cos \theta_{i}$ is the directional cosines of $\vec{B}$ with three coordinate axes.

The geomagnetic vector projection on aircraft coordinate system can be expressed as follows:

$$
\left\{\begin{array}{l}
B_{x}=B \cos \theta_{1}=B u_{1} \\
B_{y}=B \cos \theta_{2}=B u_{2} \\
B_{z}=B \cos \theta_{3}=B u_{3}
\end{array}\right.
$$

Where $\theta_{1}, \theta_{2}$ and $\theta_{3}$ are respective the angle between geomagnetic vector and $\mathrm{X}, \mathrm{Y}$ and $\mathrm{Z}$ axis.

The eddy current magnetic moment can be expressed as follows: 


$$
\left\{\begin{array}{l}
E_{x i}=e_{x i} \cdot B \cdot u_{1}^{\prime} \\
E_{y i}=e_{y i} \cdot B \cdot u_{2}^{\prime} \\
E_{z i}=e_{z i} \cdot B \cdot u_{3}^{\prime}
\end{array} \quad i=1,2, \cdots N\right.
$$

Where $\left(e_{x i}, e_{y i}, e_{z i}\right)$ is eddy current magnetic field magnetic moment coefficient, a fixed parameter, mainly depending on the geometry, quality and conductivity of magnetic material. $u_{i}^{\prime}=\left(\cos \theta_{i}\right)^{\prime}$ is the direction cosine derivative.

From the equations (1), (2), (3), (4), (5) and (6), the three-component magnetic moment can be acquired by

$$
\left\{\begin{array}{l}
M_{x i}=p_{x i}+k_{x i} \cdot B \cdot u_{1}+e_{x i} \cdot B \cdot u_{1}^{\prime} \\
M_{y i}=p_{y i}+k_{y i} \cdot B \cdot u_{2}+e_{y i} \cdot B \cdot u_{2}^{\prime} \\
M_{z i}=p_{z i}+k_{z i} \cdot B \cdot u_{2}+e_{z i} \cdot B \cdot u_{3}^{\prime}
\end{array} \quad i=1,2, \cdots N\right.
$$

As shown in Figure 1, assuming $(x, y, z)$ is the pod coordinate in the rectangular coordinate system, the magnetic dipole location coordinate is $\left(x_{0 i}, y_{0 i}, z_{0 i}\right)$ where $\mathrm{i}$ is the serial number for each dipole. The three-component magnetic field generated by helicopter at the pod can be expressed as follows

$$
\left\{\begin{array}{l}
B_{x}=\sum_{i=1}^{N}\left(M_{x i} a_{x i}+M_{y i} a_{y i}+M_{z i} a_{z i}\right) \\
B_{y}=\sum_{i=1}^{N}\left(M_{x i} b_{x i}+M_{y i} b_{y i}+M_{z i} b_{z i}\right) \\
B_{z}=\sum_{i=1}^{N}\left(M_{x i} c_{x i}+M_{y i} c_{y i}+M_{z i} c_{z i}\right)
\end{array}\right.
$$

Where

$$
\begin{array}{ll}
a_{x i}=\frac{1}{4 \pi}\left[\frac{3}{r_{i}^{5}}\left(x-x_{o i}\right)^{2}-\frac{1}{r_{i}^{3}}\right] & a_{y i}=\frac{3\left(x-x_{o i}\right)\left(y-y_{o i}\right)}{4 \pi r_{i}^{5}}=b_{x i} \\
a_{z i}=\frac{3\left(x-x_{o i}\right)\left(z-z_{o i}\right)}{4 \pi r_{i}^{5}}=c_{x i} & b_{y i}=\frac{1}{4 \pi}\left[\frac{3}{r_{i}^{5}}\left(y-y_{o i}\right)^{2}-\frac{1}{r_{i}^{3}}\right] \\
b_{z i}=\frac{3\left(y-y_{o i}\right)\left(z-z_{o i}\right)}{4 \pi r_{i}^{5}}=c_{y i} & c_{z i j}=\frac{1}{4 \pi}\left[\frac{3}{r_{i}^{5}}\left(z-z_{o i}\right)^{2}-\frac{1}{r_{i}^{3}}\right] \\
r_{i}=\sqrt{\left(x-x_{o i}\right)^{2}+\left(y-y_{o i}\right)^{2}+\left(z-z_{o i}\right)^{2}} &
\end{array}
$$

From the equations (7) and (8), it is known that when the remanence, induced magnetic moment coefficient, eddy magnetic moment coefficient and the magnetic dipole coordinates are fixed, if the directional cosines and the pod coordination are obtained, the magnetic field produced by helicopter at the pod can be solved.

\section{Simulation analysis}

The three-component direction angle of geomagnetic can be simulated by some superimposed random sine waves as follows

$$
\theta(t)=\sum_{k=0}^{n} \theta_{k} \cos \left(2 \pi f_{k} t+\varepsilon_{k}\right)
$$

Assuming the pod coordinate relative to the plane is unchanged, $\mathrm{B}=50000 \mathrm{nT}, \mathrm{N}=2,\left(x_{01}, y_{01}\right.$, $\left.z_{01}\right)=(2.0,0.5,-1.5),\left(x_{02}, y_{02}, z_{02}\right)=(1.5,0.6,1.0),\left(p_{x 1}, p_{y 1}, p_{z 1}\right)=(5 \times 104, \quad 4.2 \times 104, \quad 2.8 \times 104)$, $\left(k_{x 1}, k_{y 1}, k_{z 1}\right)=(0.6,1.3,0.9),\left(e_{x 1}, e_{y 1}, e_{z 1}\right)=(0.05,0.12,0.08),\left(p_{x 2}, p_{y 2}, p_{z 2}\right)=(1.8 \times 104, \quad 3.0 \times 104$, $4.0 \times 104),\left(k_{x 2}, k_{y 2}, k_{z 2}\right)=(1.18,0.85,1.25),\left(e_{x 2}, e_{y 2}, e_{z 2}\right)=(0.09,0.06,0.1)$, and $(x, y, z)=(5.0,1.5$, 18). 
Figure 2 shows the simulate results of helicopter background magnetic field produced at the pod.
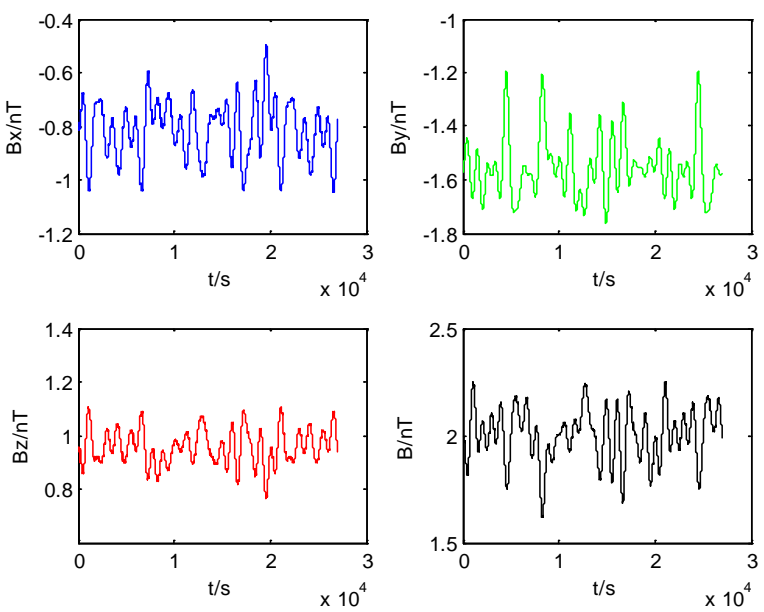

Fig.2. The helicopter magnetic interference simulate results

There are three problems need to be supplemented for above simulation process and results.

(1) The geomagnetic three-component direction cosines are obtained by three-component geomagnetic and geomagnetic total intensity, which are measured respectively by three axis fluxgate and optical pump in practical application. (2) The magnetic dipole amount, magnetic dipole position coordinates and magnetic dipole moment parameters are obtained by using the helicopter background magnetic field data measured actually to match and solve the magnetic dipole array model. (3) During flight the helicopter posture is constantly changing and the pod is affected by wind resistance, so the pod coordinates is not possible invariant.

\section{Conclusion}

It is an urgent need to develop the helicopter magnetic anomaly anti-submarine technology, there are some important problems to be solved next step:

(1) The precision of the magnetic dipole array model proposed in this paper need to be verified.

(2) There is great efforts to be made to study on the trajectory of pod under the maneuver flight of helicopter.

(3) The method of solving the magnetic dipole model parameters using experimental data should be researched.

\section{Acknowledgement}

In this paper, the research was sponsored by project ZDYZ20120102.

\section{References}

[1] Bickel S H. Small signal compensation of magnetic fields resulting from aircraft maneuvers [J]. IEEE Trans. On AES, 1979, 15(4):515-525.

[2] Zhang Changda. Airborne Tensor Magnetic gradiometric - the latest progress of airborne magnetometric technology[J].Chinese Journal of Engineering Geophysics,2006,3(5):354-361.

[3] Zhu Ziqiaug, He Jishan, Zhang Xinbing.3-Dimension Inversion of High-Precision Magnetic Data And its Geological Significance of Coal Mine Prospection[J].Journal of central south university technology,1998,29(4):307-310.

[4] Fundamentals of Naval Weapons Systems. Chapter 9 Underwater Detection and Tracking Systems[M]. Weapons and Systems Engineering Department, United States Naval Academy, 1989.

[5] Tolles W E. Compensation of induced magnetic fields in MAD equipped aircraft, OSRD 1386, sec 6. 1sr20-320[R]. [S. 1]: Airborne Instruments Lab Inc, 1943. 